\title{
Editorial
}

\section{Mathematical Approaches in Advanced Control Theories}

\author{
Baocang Ding, ${ }^{1}$ Lihua Xie, ${ }^{2}$ Weihai Zhang, ${ }^{3}$ Xianxia Zhang, ${ }^{4}$ \\ Qiang Ling, ${ }^{5}$ and Yugeng $\mathbf{X i}^{6}$ \\ ${ }^{1}$ Ministry of Education Key Lab For Intelligent Networks and Network Security (MOE KLINNS Lab), \\ Department of Automation, School of Electronic and Information Engineering, \\ $X i^{\prime}$ an Jiaotong University, Xi'an 710049, China \\ ${ }^{2}$ School of Electronic and Electrical Engineering, BLK S2, Nanyang Technological University, \\ Nanyang Avenue, Singapore 639798 \\ ${ }^{3}$ College of Information and Electrical Engineering, Shandong University of Science and Technology, \\ Qingdao 266590, China \\ 4 Shanghai Key Laboratory of Power Station Automation Technology, School of Mechatronics Engineering \\ and Automation, Shanghai University, Shanghai 200072, China \\ ${ }^{5}$ Department of Automation, University of Science and Technology of China, Hefei 230026, China \\ ${ }^{6}$ Department of Automation, Shanghai Jiaotong University, Shanghai 200240, China
}

Correspondence should be addressed to Baocang Ding, baocang.ding@gmail.com

Received 20 September 2012; Accepted 20 September 2012

Copyright (C) 2012 Baocang Ding et al. This is an open access article distributed under the Creative Commons Attribution License, which permits unrestricted use, distribution, and reproduction in any medium, provided the original work is properly cited.

Advanced control theory fills a gap between the mathematical control theory and modern control engineering practices. Conceptually, advanced control theories can include any theoretical problems related to the controller design. But in this issue it may include model predictive control, sliding mode control, robust control, real-time optimization, and identification and estimation, which are not limited to controller design. Advanced control technologies have become ubiquitous in various engineering applications (e.g., chemical process control, robot control, air traffic control, vehicle control, multiagent control, networked control). The development of mathematical methods is essential for the applications of advanced control theories. Sometimes, it lacks effective methods to tackle the computational issue (e.g., model predictive control of a fast process). Sometimes, a new application requires a brand-new solver for applying the advanced control theory (e.g., a new production line far exceeding the usual speed). The main focus of this special issue will be on the new research ideas and results for the mathematical problems in advanced control theories.

A total number of 63 papers were submitted for this special issue. Out of the submitted papers, 25 contributions have been included in this special issue. The 25 contributions consider several closely related and interesting topics. 
The subjects in controller design and synthesis have occupied 13 contributions. These contributions include, for example, variable structure control, model predictive control (MPC), fuzzy logic control (FLC), robust control, networked and distributed control, optimal control, and adaptive control. For Markovian jump nonlinear systems with unmodeled dynamics and Wiener noise, J. Zhu et al. use backstepping technique and stochastic smallgain theorem, so as to design a switch controller such that stochastic stability is guaranteed. L. Ding et al. consider sliding mode control for mobile manipulators with stochastic jump switching joints. Adaptive parameter techniques are adopted to cope with the effect of Markovian switching and nonlinear dynamics uncertainty and follow the desired trajectory for wheeled mobile manipulators. T. Zou considers the dynamic stability property of the double-layered MPC. The double-layered MPC is a common structure for real applications. $X$. Zhang et al. develop a three-dimensional FLC design methodology based on clustering and support vector machine regression learning from a spatiotemporal dataset. Y. Wang focuses on the robust stability of Lotka-Volterra predator-prey system with the fuzzy impulsive control. Y. Zhu and L. Fan present the robust force/motion control strategies for mobile manipulators under both holonomic and nonholonomic constraints in the presence of uncertainties and disturbances. Y. H. Yang and C. L. Chen propose a generic spatial domain control scheme for a class of nonlinear rotary systems of variable speeds with spatially periodic disturbances. When the system state is unmeasurable, a nonlinear state observer is established for providing the estimated states. Q. Ling et al. investigate the effects of the network-induced delays, packet dropouts, and the torque disturbance on the speed tracking of a permanent magnet synchronous motors system. The designed controller can robustly guarantee stability and performance. Q. Ling and H. Deng study the second moment stability of a Markov jump linear system with real states. They propose an alternative necessity proof of the stability condition for this system. Y. Guo and T. Pan investigate the problem of robust stability of uncertain linear discrete-time system over network with bounded packet loss. X. Liu et al. consider a containment problem of networked fractional-order system with multiple leaders under a fixed directed interaction graph. The distributed protocol, flocking problem, communication delay, and interconnection topology are studied. Y. Lei et al. establish an optimal control model of distributed parameter systems (DPS) for polymer injection strategies. G. M. Bahaa considers the optimal boundary control problem for an infinite order distributed parabolic systems with multiple time delays given in the integral form both in the state equations and in the Neumann boundary conditions.

Closely related to the controller design and synthesis are the 8 contributions on the estimation problem. These contributions include, for example, Kalman filter, robust state estimator, fusion estimator, target tracking filter, and modeling parameter estimation. X. Lu et al. deal with Kalman smoothing problem for wireless sensor networks with multiplicative noises. Packet loss occurs in the observation equations, and multiplicative noises occur both in the system state equation and the observation equations. L. Zhang et al. revisit the state estimator for polytopic uncertain systems. The notion of quadratic boundedness, which has been useful in specifying stability of the system with uncertain but bounded noise, is utilized. H. Zhao and C. Zhang consider the finite-time $H_{\infty}$ filter for linear continuous time varying systems with uncertain observations and $\mathcal{L}_{2}$-norm bounded noise. By using the projection theory in Krein space, the finite-time $H_{\infty}$ filter is solved. X. Wang and S. Sun present a self-tuning weighted measurement fusion Kalman filter, for the linear discrete stochastic systems with multiple sensors and unknown noise statistics. It is proved that the presented self-tuning weighted measurement fusion Kalman filter converges to the optimal 
weighted measurement fusion Kalman filter. F. Lian, C. Han et al. study the convergence of the Gaussian mixture extended-target probability hypothesis density filter and its extended Kalman filtering approximation in mildly nonlinear condition. The problem of extendedtarget tracking is very valuable for many real applications, such as ground or littoral surveillance, robotics, and autonomous weapons. F. Lian, C. Li et al. study the convergence for the sequential Monte Carlo implementations of the multitarget multi-Bernoulli (MeMBer) filter and cardinality-balanced MeMBer filters. By combining the linear matrix inequalities for the system identification and those to obtain a discrete time controller, K. Hiramoto proposes a framework to integrate two steps for the model-based control system design, that is, the SISO system identification and the controller synthesis. K. Kobayashi and K. Kaito utilize a one-dimensional consolidation model, incorporating inhomogeneous ground subsidence, to generate the sets of sample paths designating ground subsidence processes. The estimation of ground subsidence processes is an important subject for the asset management of civil infrastructures on soft ground, such as airport facilities.

There are also 3 contributions on the image processing and acoustic signal processing. These contributions can be seen as the extensions of the estimation problem, in the context of this special issue. $\mathrm{L} . \mathrm{Zi}$ and J. Du propose an energy-driven image interpolation algorithm employing Gaussian process regression. Image interpolation, as a method of obtaining a high-resolution image from the corresponding low-resolution image, is a classical problem in image processing. P. Han and J. Du use a nonsubsampled pyramid structure and a nonsubsampled directional filter to achieve multidimensional and translation-invariant image decomposition for spatial images. Spatial images are inevitably mixed with different levels of noise and distortion. C. Wang and P. Zhang present an improved spoken term detection strategy, which integrates a phoneme confusion matrix and an improved word-level minimum classification error training method. Spoken term detection system will degrade significantly if there is mismatch between acoustic model and spontaneous speech.

The last contribution is for the mathematical programming. T. Hasuike et al. consider the risk-control and management approach for a bottleneck spanning tree problem under the situation where edge costs in a given network include randomness and the reliability. Note that several other contributions mentioned above have also considered optimizations (e.g., in the work of G. M. Bahaa, necessary and sufficient optimality conditions for the Neumann problem with the quadratic performance functional are derived; in the work of Y. Lei et al., the necessary conditions for optimality are obtained through application of the calculus of variations and Pontryagin's weak maximum principle; in the work of X. Wang and S. Sun, the global optimality property is considered; some other works utilize optimization to obtain the controller, estimator, or modeling parameters).

From the above contributions, some trends in the advanced control theories seem to become clearer. The studied problems are becoming more and more complex (e.g., considering disturbance/noise, stability/convergence, probability/randomness, distributed parameters, time-delay/networked phenomenon, physical constraints, impulsive system, optimization and control integrated system, fractional-order system, and parametric uncertainties). Usually, several kinds of complexities should be included into a single work in order for it to be publishable as a contribution.

We hope the readers of this special issue will find it interesting and stimulating and expect that the included papers to contribute to further advance the area of advanced control. Finally, we would like to thank all the authors who have submitted papers to the special issue and the reviewers involved in the refereeing of the submissions. 
Journal of Applied Mathematics

Baocang Ding Lihua Xie

Weihai Zhang

Xianxia Zhang Qiang Ling Yugeng Xi 


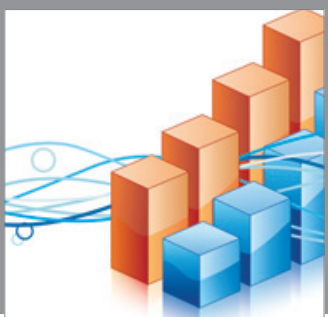

Advances in

Operations Research

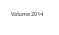

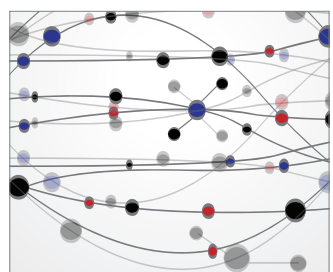

\section{The Scientific} World Journal
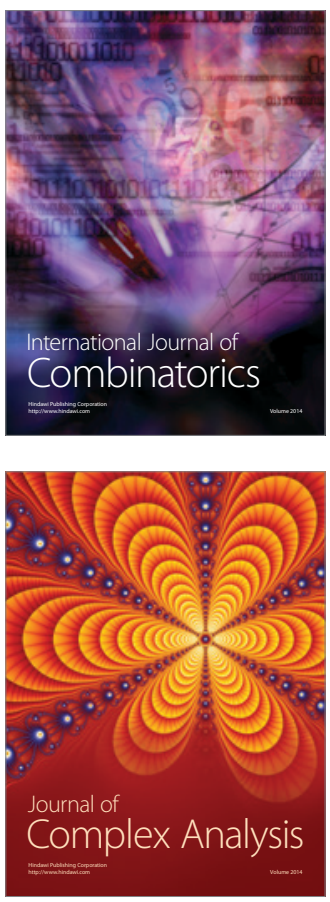

International Journal of

Mathematics and

Mathematical

Sciences
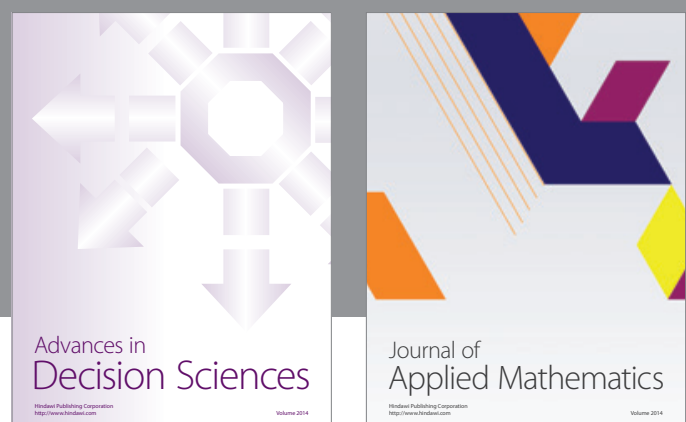

Journal of

Applied Mathematics
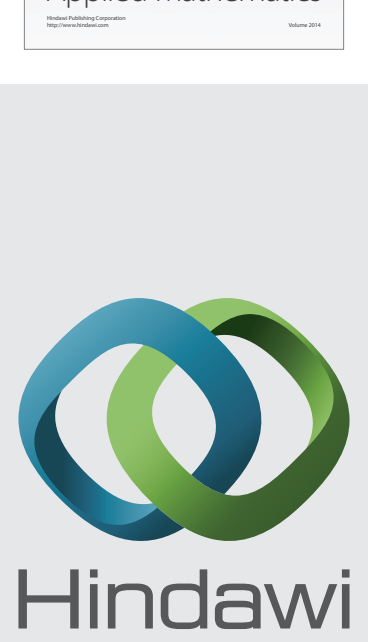

Submit your manuscripts at http://www.hindawi.com
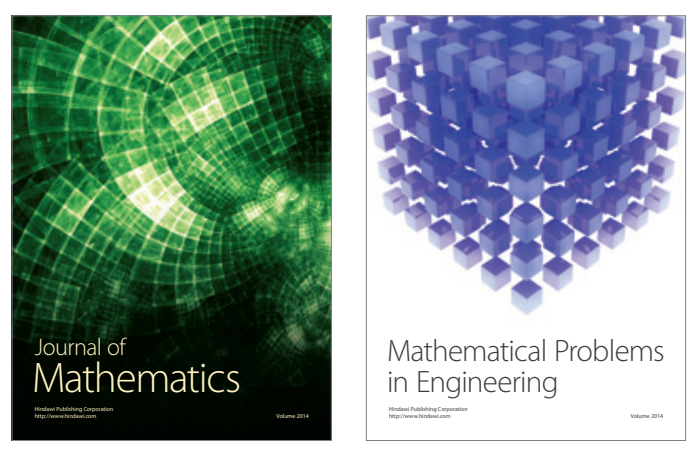

Mathematical Problems in Engineering
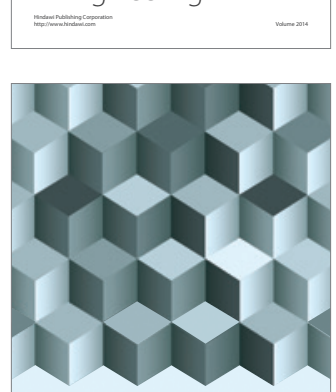

Journal of

Function Spaces
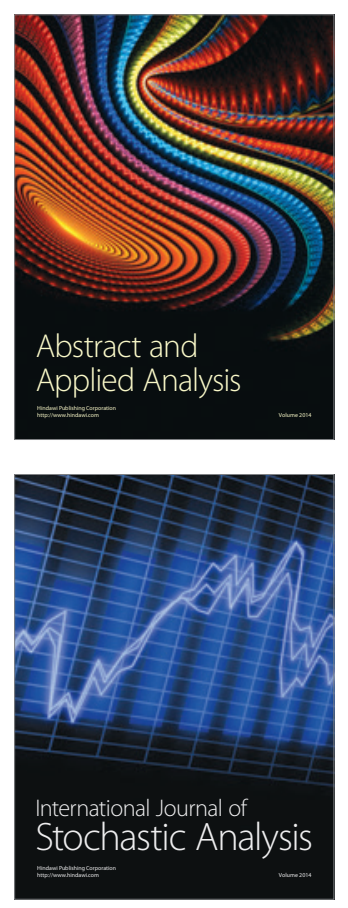

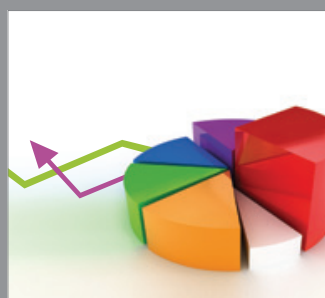

ournal of

Probability and Statistics

Promensencen
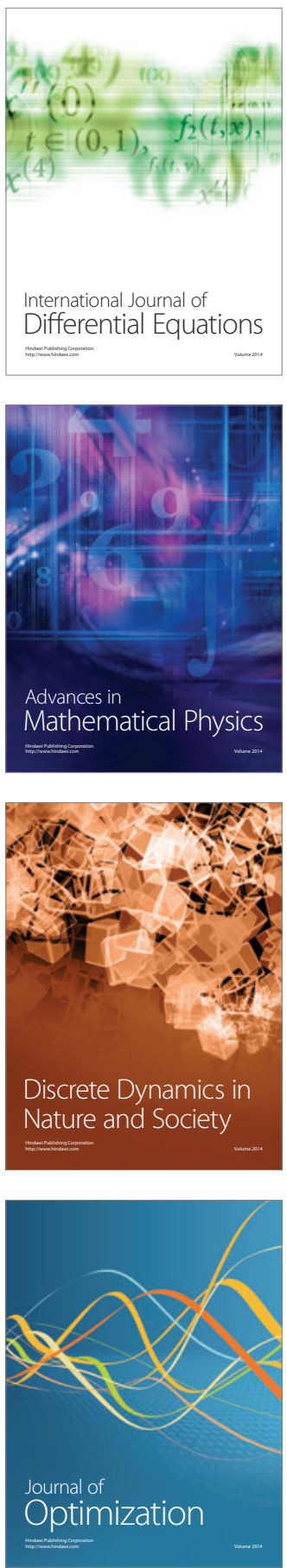